\title{
DIAGNOSTIC DILEMMA OF AN ADNEXAL MASS: GIANT BROAD LIGAMENT FIBROID
}

Hrishikesh M. Mandhane ${ }^{1}$

\section{HOW TO CITE THIS ARTICLE:}

Hrishikesh M. Mandhane. "Diagnostic Dilemma of an Adnexal Mass: Giant Broad Ligament Fibroid". Journal of Evolution of Medical and Dental Sciences 2015; Vol. 4, Issue 17, February 26; Page: 2975-2978,

DOI: $10.14260 /$ jemds/2015/429

ABSTRACT: Giant fibroids are known to arise from the uterus, but rarely from the broad ligament also. This is a case report of a giant broad ligament fibroid, reported for its rarity, and the diagnostic difficulties.

KEYWORDS: Giant broad ligament fibroid, uterine fibroids.

INTRODUCTION: Extrauterine fibroids though do occur, but are not common as uterine fibroids. Among the extrauterine fibroids, broad ligament fibroids are the most common to occur. ${ }^{1}$ although its overall incidence being rare. Because of its rarity it poses specific diagnostic difficulties causing an error in making the final diagnosis and therefore the management. This is one such case report where a diagnosis of malignant ovarian tumour was made and the histopathology confirmed the diagnosis of broad ligament fibroid.

CASE REPORT: Forty-eight years old para 3, living 3, came to OBGY department of PDDVPF \& Hospital with the history of distension of abdominal for six months that rapidly increased over the past 1 months. Along with this she had difficulty in urination since 1 month. There was history of indigestion. No history of weight loss/ anorexia/ fever. She was a postmenopausal female.

EXAMINATION: A middle aged lady of average build and nutrition. No pallor. BP 120/70 mm Hg, Pulse 90bpm, RR 12/minute. No significant lymphadenopathy. Systemic examination was normal. Abdomen was distended. A firm, smooth surfaced, regular, non-tender mass of approximately 22 weeks size was felt arising out of the pelvis. With side to side mobility present. Lower margin of the mass could not be reached. Two separate masses could be palpated but could not be moved separately. No shifting dullness. Speculum examination revealed normal cervix and vagina. Per vaginum examination cervix was normal to feel, regular. Uterus was around 10 week's size. anteverted, mobile and non-tender. A firm to hard, non-tender mass was felt in continuity of uterus. Per rectal examination was normal.

INVESTIGATIONS: Hemoglobin 11.6 gm\%, Blood Urea $24 \mathrm{mg} / \mathrm{dl}$, Serum Creatinine $0.8 \mathrm{mg} / \mathrm{dl}$, Random blood sugar $100 \mathrm{mg} / \mathrm{dl}$, Na-140 K-4 Cl-100 Tco2-25, Urine c/s- no growth. Ultrasonography of the abdomen: a $179 \times 125 \times 68 \mathrm{~mm}$ well defined heterogeneous mass with cystic areas within it and extending above umbilicus. Uterus is visualized separately from mass and displaced superiorly. There is a $66 \times 44 \mathrm{~mm}$ posterior wall fibroid. No e/o free fluid. Impression: S/0 neoplasic etiology of ovarian origin.

MANAGEMENT: With a probable diagnosis of malignant ovarian tumor the patient was taken up for an exploratory laparotomy. 


\section{CASE REPORT}

OPERATIVE FINDINGS: No evidence of ascetic fluid. A mass of about $25 \times 25 \mathrm{~cm}$ identified on opening of abdomen. Mass was adherent on posterior aspect and on right. Mass was separated of its adhesions and was exteriorized out of cavity. Followed by two separate masses were dissected both with sharp and blunt dissection taking care of safety of the Ureter. Mass separated from uterus and delivered, B/L ovaries appeared normal followed by hysterectomy and B/L oophorectomy was done. Rest of the abdominal organs was normal. Sutures were removed on the 10th postoperative day. Patient was discharged in a satisfactory condition. Intra-operative view (Fig. 1)

PATHOLOGY: Gross - Mass $15 \mathrm{cms}$ x $14 \mathrm{cms}$ and weighing 1450 gms. External surface smooth. Cut section shows predominantly solid areas along with cystic areas containing yellowish gelatinous substance and altered hemorrhagic fluid. Uterus $9 \mathrm{cms}$ x $7 \mathrm{cms} \times 4 \mathrm{cms}$. Cervix normal. Posterior wall of uterus showing intra myometrial fibroid. External surface over the fibroid shows dilated prominent blood vessels. On gross appearance it looked (Fig. 2).

MICROSCOPIC: Mass showed benign spindle cell tumour of the stroma tissue origin with marked calcificed degeneration -Leiomyoma of the broad ligament (Fig. 3).

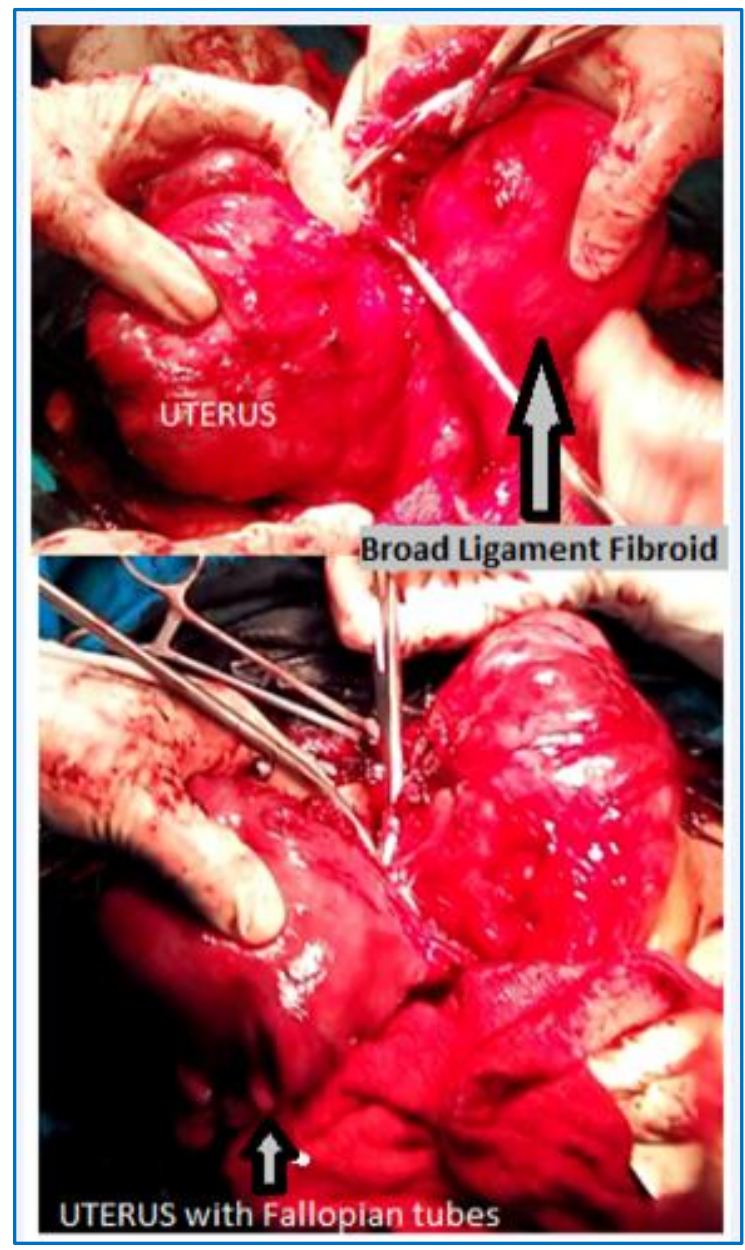

Fig. 1: Intra operative appearance. 


\section{CASE REPORT}

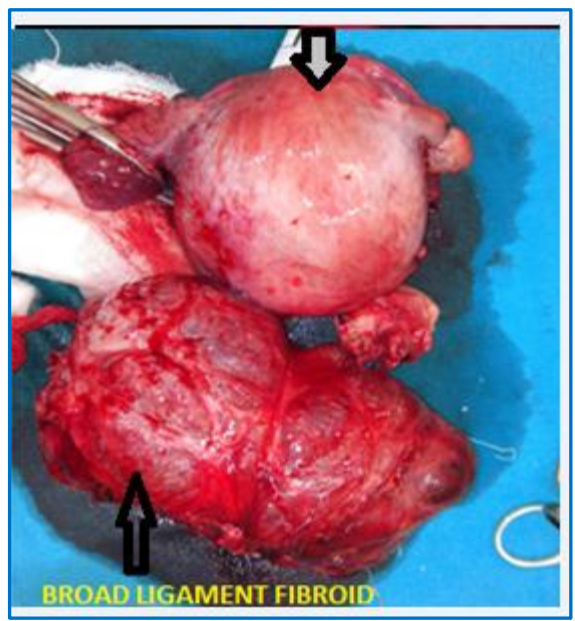

Fig. 2: Gross Specimen of

Broad Ligament Fibroid

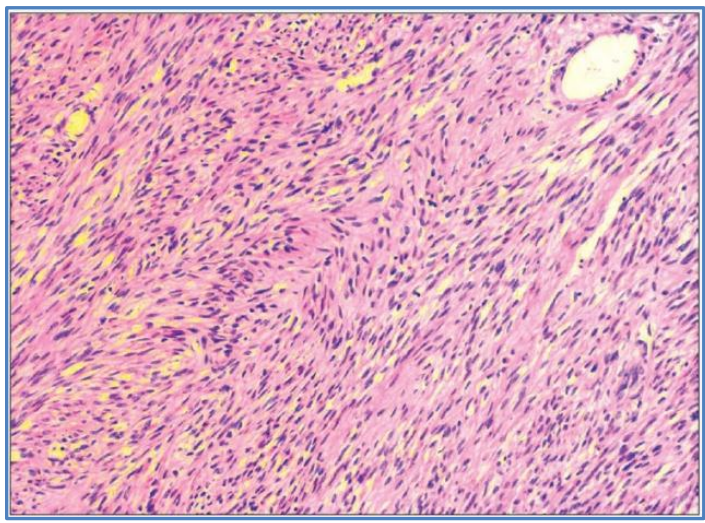

Fig.3: Histopathology of

Broad Ligament Fibroid

DISCUSSION: Uterine leiomyomas, also known as myomas or fibroids, are by far the commonest benign uterine tumours. They are usually diagnosed on physical examination. They are most commonly intramural, subserosal, submucosal and cervical. Myomas and fibromyomas are not uncommon in the round, ovarian and broad ligaments. ${ }^{2}$ They are found in association with similar uterine tumours and their pathology and complications are the same as fibroids. Fibroids in the broad ligament though not so common, but are well known for achieving enormous size; which may mimic a malignancy of the pelvis thereby altering the course of treatment offered.

This case reports one such incidence where the patient presented with a mass per abdomen that seem to be arising out of the pelvis, had features of malignancy both clinically as well as radiologically and received treatment for the same while histopathology confirmed it as a benign broad ligament fibroid. Had it been diagnosed initially the major debulking surgery could be limited to a simple hysterectomy. A similar case report has also been reported by Gowri et al. ${ }^{3}$ For its rarity, and the diagnostic difficulties it posed. 
Degenerative changes in the leiomyoma as are considered to be due to inadequate blood supply and degenerative changes seem to depend on the degree and rapidity of the onset of vascular insufficient changes are the commonest form of degeneration. The fibroid in our report had undergone calcific degeneration. Bose et al have also reported a similar case of calcified broad ligament fibroid. ${ }^{4}$ There is no particular relationship between any symptom or group of symptoms and the incidence of degenerative changes.

CONCLUSION: The broad ligament benign tumour even though being uncommon can grow to a large size as exemplified in this case. Degenerative changes occur even in such extra-uterine fibroids.

\section{REFERENCES:}

1. Bhatla N. Tumours of the corpus uteri. In: Jeffcoats Principles of Gynaecology 6th edn. Arnold Printers, London; 2001: pp 470.

2. Jonathan S Berek. Benign Diseases of the Female Reproductive Tract. In: Novak's Gynecology 13th edn. Lippincott Williams \& Wilkins, Philadelphia; 2002: pp 380.

3. Gowri V, Sudheendra R, Oumachigui A, Sankaran V. Giant broad ligament leiomyoma. Int J Gynaecol Obstet 1992; 37(3): 207-10.

4. Bose GK, Saha SK, Adhikari S, Chatterjee S. An unusual case of calcified broad ligament fibroid. J Obstet Gynecol Ind 1996; 46(3): 435-36.

\section{AUTHORS:}

1. Hrishikesh M. Mandhane

\section{PARTICULARS OF CONTRIBUTORS:}

1. Senior Resident, Department of Obstetrics \& Gynaecology, Padmashree Dr. Vithalrao Vikhe Patil Medical College \& Hospital.

FINANCIAL OR OTHER COMPETING INTERESTS: None

\section{NAME ADDRESS EMAIL ID OF THE} CORRESPONDING AUTHOR:

Dr. Hrishikesh M. Mandhane,

Mandhane Hospital,

Court Road,

Paithan, Aurangabad-431107.

E-mail: hrishmmandhane@gmail.com

Date of Submission: 24/01/2015.

Date of Peer Review: 27/01/2015.

Date of Acceptance: 17/02/2015.

Date of Publishing: 25/02/2015. 